\title{
Uma Discussão sobre Ambientes Virtuais 3D como Suporte ao Estudo da Arquitetura
}

\author{
Maria Amelia Eliseo ${ }^{1}$, Darlan Silva Alves Delmondes ${ }^{1}$, Luiz Henrique Falavigna ${ }^{2}$, \\ Jorge Henrique Garcia ${ }^{1}$
}

${ }^{1}$ Faculdade de Computação e Informática - Laboratório de Processamento Gráfico Universidade Presbiteriana Mackenzie

São Paulo, SP - Brazil

${ }^{2}$ Faculdade de Arquitetura e Urbanismo - Universidade Presbiteriana Mackenzie São Paulo, SP - Brazil

mamelia@mackenzie.br, darlan.dsad@gmail.com, \{luiz_falavigna,

j.hrique\} @hotmail.com,

\begin{abstract}
Three-dimensional virtual environments have brought new interaction experiences to the users. The immersion sensation provided by these environments is a way of motivation to learning that leads to more involvement and better understanding of three-dimensional space. With computer graphics and semantic resources, it is possible to depict virtually different moments of buildings with historical significance providing a spatiotemporal interaction and visualization. This paper discusses the potential of these environments that depict buildings with historical significance as supporting subjects that consider studies on the Theory and History of Architecture.
\end{abstract}

Resumo. Os ambientes virtuais tridimensionais têm trazido novas experiências de interação para os usuários. A sensação de imersão proporcionada por tais ambientes é uma forma de motivação ao aprendizado que leva a maior envolvimento e melhor entendimento do espaço tridimensional. Com recursos de computação gráfica e semântica é possível retratar virtualmente diferentes momentos de edifícios com significados históricos, proporcionando uma interação e visualização espaço-temporal. Este artigo discute o potencial destes ambientes que retratam edificações com significado histórico como suporte a disciplinas que contemplam estudos voltados para a Teoria e História da Arquitetura.

\section{Introdução}

Os arquitetos se utilizam de ferramentas de desenho 2D como plantas, cortes, fachadas e perspectivas para comunicar e demostrar o espaço arquitetônico tridimensional. Mas, estas formas de representação possuem algumas limitações e nem sempre fazem transparecer aquilo que está sendo reproduzido. Para interpretar estes desenhos técnicos e compreender de fato o espaço, é preciso adquirir conhecimento e prática na leitura destas peças gráficas. $\mathrm{O}$ aluno que está ingressando num curso de arquitetura, num primeiro momento se depara com a dificuldade de interpretação destes desenhos. 
Além disso, a arquitetura em sua essência, que tem o ser humano como integrante e protagonista do espaço, inclui informações que nem sempre são transmitidas através do desenho, como valores culturais, sociais ou econômicos associados ao contexto do edifício [Zevi 2002]. Assim, a representação gráfica através da perspectiva, plantas, cortes e fachadas embora pareça uma representação adequada para a arquitetura, não é suficiente para mostrar esta essência. Para que a arquitetura seja representada em sua plenitude estas partes devem ser consideradas.

Neste contexto, os ambientes virtuais tridimensionais têm sido empregados com sucesso para estudar, comunicar e representar o projeto de arquitetura [Maze 2002]. Os recursos da computação gráfica propiciam a reconstrução digital de espaços arquitetônicos tridimensionais aprimorando a visualização e compreensão da arquitetura, inclusive reproduzindo ambientes que dão a sensação de imersão, ou seja, o usuário se sente, mesmo que psicologicamente, como parte integrante do ambiente virtual. Utilizando-se destes recursos e com o intuito de preservar a memória de edificações e sítios arqueológicos considerados relevantes para a história, surgiram aplicações que retratam reconstruções digitais tridimensionais, reproduzindo, num ambiente virtual, espaços arquitetônicos que simulam o real. Muitas destas aplicações permitem ao usuário explorar os espaços internos e externos do edifício com interação através de mouse e teclado ou luvas de dados [Frischer 2005], [Roussou 2000], [Rome Reborn 2010], [Cabral et al. 2007] e [Bernardes 2002].

A associação de descrições semânticas aos elementos gráficos, gerados com os recursos da computação gráfica, permite estender a visualização do ambiente virtual complementando-a com informações textuais, além de construir relacionamentos inerentes a transições espaço-temporais e a pluralidade dos valores da arquitetura [Eliseo 2012]. A reconstrução digital de um espaço arquitetônico com transições espaço-temporais, acrescido de descrições semânticas se constitui num ambiente propício para representar e descrever edifícios com significado histórico, podendo ser utilizado para aprimorar o aprendizado de disciplinas que contemplam estudos voltados para a Teoria e História da Arquitetura.

Este artigo discute o potencial dos ambientes virtuais tridimensionais com interação e visualização espaço-temporal complementados com descrições semânticas. A intenção é mostrar que estes ambientes oferecem suporte a estudos que envolvam a compreensão do espaço arquitetônico, especialmente disciplinas como a Teoria ou História da Arquitetura, uma vez que provoca a sensação de imersão e envolvimento no aprendiz, permitindo, através da interação, explorar, não apenas a tridimensionalidade, mas outros fatores como valores históricos, culturais e sociais envolvidos na representação.

Também mostra a implementação de um ambiente virtual tridimensional de um espaço arquitetônico associado a uma ontologia espaço-temporal para as descrições semânticas, que foi exposto num Centro Histórico para visitação. Nesta oportunidade os usuários interagiram com a aplicação e em seguida responderam um questionário que avaliava as sensações de imersão, envolvimento e realidade, a validade de comunicação das informações semânticas e a usabilidade. Os resultados da avaliação são apresentados no final deste artigo. 


\section{Ambientes Virtuais e o Aprendizado na Arquitetura}

Um curso de Arquitetura é fundamentado no processo criativo do projeto do espaço arquitetônico, enfatiza o lado prático nas disciplinas que o compõem, destacando como os edifícios são feitos e como os componentes tectônicos são fundamentais para definir características da arquitetura. Prioriza o desenho e as habilidades artísticas e estéticas, que são aplicadas em técnicas de representações gráficas, construções de maquetes e modelos, etc., além das disciplinas teóricas que discutem história, cultura, aspectos sociais, sustentabilidade, materiais, técnicas construtivas.

Uma das habilidades dos alunos do curso de Arquitetura é a percepção e a compreensão do espaço volumétrico tridimensional, representado por desenhos técnicos em duas dimensões (plantas, cortes e fachadas). Foi realizado um experimento com estudantes de arquitetura para investigar e comparar a compreensão do volume e espaço em três diferentes condições de representação [Schnabel e Kvan 2003]. O procedimento reproduz um processo de projeto arquitetônico no qual volumes e espaços são determinados, a fim de gerar uma concepção global, a partir da montagem de cubos. A primeira condição envolveu a representação convencional do espaço $3 \mathrm{D}$ a partir dos tradicionais desenhos técnicos 2D. A segunda e terceira condições empregaram modelos 3D digitais: a segunda utilizou um ambiente virtual com interação e manipulação a partir do monitor do computador e a terceira, um ambiente virtual imersivo. Embora os estudantes da primeira condição tenham atingido o maior grau de precisão na reconstrução do volume, eles não entenderam completamente o arranjo dos cubos dentro do volume. Já os alunos que trabalharam com os dois ambientes virtuais exploram e investigaram a forma e as relações espaciais de uma forma mais holística e, portanto, tiveram um melhor entendimento da tridimensionalidade do volume e seus componentes.

Nos ambientes virtuais imersivos os usuários interagem com os objetos virtuais de forma mais próxima do real, abrindo possibilidades ao trabalho colaborativo e participativo, por exemplo, realizando uma discussão entre os envolvidos que irão frequentar o novo edifício. Com isso, trabalhadores e clientes, que irão utilizar o novo edifício podem fazer um passeio virtual imersivo, ainda em fase de projeto, para avaliar a qualidade dos espaços propostos junto com a equipe de arquitetos [Mobach 2008].

Em disciplinas teóricas como Teoria e História da Arquitetura, os alunos analisam a produção arquitetônica e urbanística ocorridas em diferentes períodos, bem como suas relações com os aspectos de ordem social, econômica, política e cultural. Os ambientes virtuais imersivos oferecem um melhor entendimento volumétrico do espaço, provocam a sensação de imersão e envolvimento e fornecem possibilidades de exploração visual e textual. Esses se constituem em ferramentas que auxiliam a compreensão da produção arquitetônica e urbanística.

\section{Um Ambiente Virtual Tridimensional Associado a uma Ontologia Espaço- Temporal}

Um ambiente virtual tridimensional implica na modelagem gráfica através da representação geométrica dos elementos gráficos $3 \mathrm{D}$, as primitivas $3 \mathrm{D}$ e a ferramenta matemática para representar e visualizar os objetos, além da renderização, responsável pelos modelos de iluminação, luz, sombra, reflexo, cores e texturas [Gutiérrez, Vexo e 
Thalmann 2008]. Trata-se de ambiente sintético, gerado por computador, composto por elementos gráficos onde o usuário tem a sensação de imersão.

Foi escolhido o Edifício Mackenzie pelo seu significado histórico para ser representado digitalmente e constituir o ambiente virtual tridimensional. A escolha levou em consideração o tombamento realizado pelo CONDEPHAAT (Conselho de Defesa do Patrimônio Histórico, Artístico, Arqueológico e Turístico do Estado de São Paulo), que lhe concedeu o grau de preservação 1, ou seja, tanto as partes externas quanto internas devem ser preservadas. O edifício escolhido foi tombado como bem cultural de interesse histórico-arquitetônico não só pela tipologia arquitetônica, mas também pelo significado histórico-cultural que representou em relação ao ensino [São Paulo (Estado) 1993].

A escolha deste edifício abre possibilidades de representação, não apenas gráfica, mas também de seus valores sociais e culturais. Por isso, uma ontologia espaçotemporal foi associada aos elementos gráficos que o representam para estabelecer relacionamentos vinculados à sua história e às suas transformações espaciais ocorridas desde sua construção.

Uma ontologia é a representação do conhecimento de um domínio do discurso e consiste de uma lista de termos e relacionamentos entre estes termos. Descreve formalmente os termos que denotam conceitos importantes, onde um conjunto de objetos e seus relacionamentos são apresentados em forma de vocabulário, como classes de objetos do domínio. As relações incluem hierarquias de classes. As ontologias podem incluir informações como propriedades, restrições, declarações disjuntas, especificações de relacionamentos lógicos entre objetos [Antoniou e Van Harmelen 2008] [Breitman et al. 2007]. O propósito da ontologia é caracterizar uma conceitualização, limitando as possibilidades de interpretação de símbolos não lógicos de uma linguagem lógica, de modo a estabelecer um consenso sobre o conhecimento descrito [Guarino 1998].

A ontologia elaborada descreve conceitos no domínio da arquitetura, considerando as transformações ocorridas no espaço arquitetônico ao longo do tempo e seus relacionamentos espaço-temporais [Eliseo 2011]. Descreve o estado de conservação do edifício e as possíveis transformações ocorridas no seu espaço físico relacionadas com o momento ou período de tempo em que ocorreram e os motivos que as ocasionaram. Também estabelece um relacionamento com os materiais construtivos utilizados. Vale ressaltar que essa ontologia foi construída de forma flexível para incorporar descrições de outros espaços arquitetônicos. Após implementada na linguagem RDF (Resource Description Framework) foi utilizada para a inserção de descrições semânticas nos elementos gráficos, que tornam-se únicos, pois definem características específicas daquele objeto, que poderá ser reutilizado em outros cenários ou aplicações. A inserção foi realizada através do framework Jena, desenvolvido em Java, para extrair as informações solicitadas para a descrição da cena.

Os elementos gráficos foram construídos com a linguagem de marcação X3D (Extensible 3D), formato padrão para representar cenas e objetos 3D usando a linguagem XML, acrônimo de eXtensible Markup Language [Web3D Consortium 2012]. Por ser baseado em XML, a linguagem oferece suporte à inclusão das descrições semânticas nos elementos gráficos. Também pode ser embutida na linguagem HTML 5 
(HyperText Markup Language) permitindo a transmissão do ambiente virtual através da Internet, incluindo a portabilidade para dispositivos móveis.

Para integrar uma cena X3D com a HTML 5 é necessário utilizar o framework X3DOM, que faz a inclusão de elementos X3D como parte da árvore DOM (Document Object Model) da HTML5. Assim, é possível manipular o conteúdo 3D adicionando, alterando ou removendo os elementos DOM HTML e dispensar o uso de plug-ins externos para visualizar o conteúdo 3D [Behr et al. 2009].

Com esta abordagem, foi feita uma reconstrução digital tridimensional do edifício escolhido, de forma que o usuário escolha um período no tempo, percorra seus espaços e encontre informações textuais que complementam a visualização durante seu "passeio virtual". Estas informações são fornecidas pela ontologia incluída nos elementos gráficos. A Figura 1 mostra o cenário do edifício visualizado a partir de um navegador web, no ano de 1896, época da inauguração do edifício. No canto superior esquerdo da Figura 1 aparece uma informação textual que é exibida quando o usuário se depara com a escada.

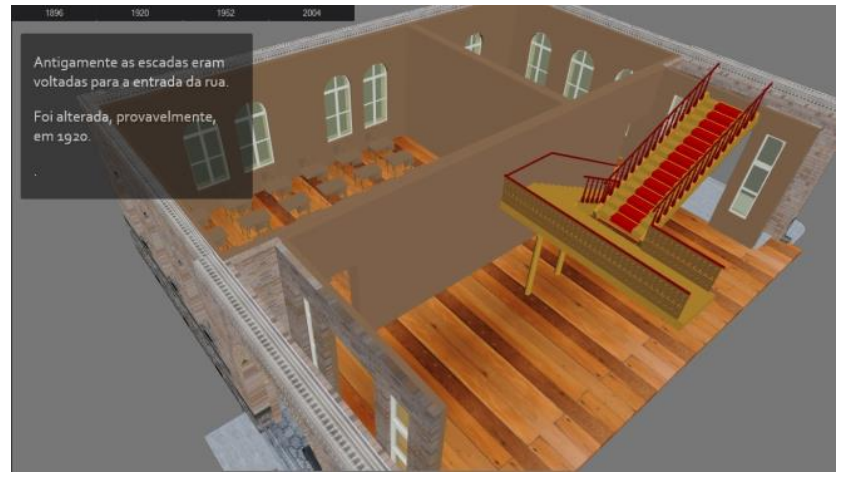

Figura 1. Cena do edifício virtual com interação espaço-temporal acrescida de descrições semânticas

Este mesmo ambiente virtual tridimensional foi utilizado em uma exposição num Centro Cultural. Aproveitando a flexibilidade oferecida pela sua implementação e utilizando de uma webcam foi realizado um experimento fazendo uso dos recursos de realidade misturada, ou seja, a mistura dos ambientes real e virtual. A reconstrução digital do edifício foi projetada na parede. Ao entrar na sala onde havia a projeção, a webcam detectava a presença do visitante, em tempo real, fazia o reconhecimento do corpo da pessoa e integrava a nova imagem à cena 3D (Figura 2).

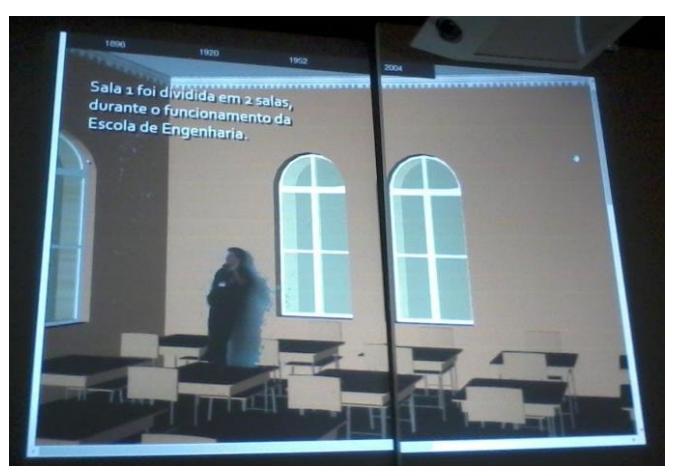

Figura 2. Integração do ambiente virtual tridimensional com a imagem real do visitante 
Com a intenção de avaliar a experiência e a satisfação do usuário ao interagir com o ambiente virtual tridimensional projetado na parede, foi elaborado um questionário aplicado aos visitantes, baseado em outros dois já existentes: o IPQ ("Igroup Presence Questionnaire") e o QUIS ("Questionnaire for User Interaction Satisfaction").

O IPQ busca medir a sensação de presença do usuário em ambientes virtuais tridimensionais, através de escalas de medição subjetivas, um a vez que esta difere da medição do grau de imersão, que é mensurado objetivamente. O questionário divide a avaliação em três fatores básicos [Igroup Project Consortium 2008]:

- Presença espacial: é a sensação de estar físicamente presente no ambiente virtual;

- Envolvimento: mede a atenção aplicada no ambiente virtual e o envolvimento;

- Realismo: mede o quão o ambiente foi realista.

O QUIS, elaborado pelo Human-Computer Interaction Lab (HCIL) da Universidade de Maryland [Harper e Norman 1993], foi construído para avaliar a satisfação subjetiva dos usuários sobre aspectos específicos de interface humanocomputador (IHC): fatores da tela, terminologia e sistema de feedback, fatores de aprendizagem, capacidades do sistema, manuais técnicos, tutoriais online, multimídia, teleconferência, e a instalação do software.

O questionário elaborado utilizou a escala Likert, que mede o grau de concordância ou discordância sobre algo escolhendo um ponto numa escala com gradações. Avaliou as sensações de presença, envolvimento e realismo, a eficiência das informações textuais e a usabilidade do ambiente. Os resultados desta avaliação são mostrados na próxima seção.

\section{Avaliação dos Usuários e Resultados}

A reconstituição tridimensional do edifício foi implantada num Centro Histórico que recebeu visitantes para interagirem com o ambiente virtual tridimensional. Após a interação, os usuários foram convidados a responderem o questionário de avaliação. Responderam ao questionário 41 participantes acostumados a utilizar tecnologias digitais, incluindo o uso de dispositivos móveis em suas tarefas cotidianas. A maioria eram estudantes dos cursos de computação e arquitetura. Além disso, $85 \%$ não tiveram experiências anteriores com interação em ambientes virtuais tridimensionais imersivos.

A questão que avaliou a sensação de presença ("Eu me senti presente na sala daquela época"), mostrou que $70,7 \%$ dos participantes concordaram com a afirmação, $29,3 \%$ responderam indiferente e ninguém discordou. Isto demonstra que, apesar de ser um ambiente virtual, o mesmo conseguiu estabelecer um estímulo de presença entre o participante e o cenário.

Sobre o grau de realismo oferecido pelos experimentos, medido na questão "Quão real o passeio na sala the pareceu?", 19,5\% dos participantes consideraram os experimentos nada real, $53,7 \%$ dos respondentes se demonstraram indiferentes e $24,4 \%$ 
afirmaram que passeio foi real. Uma pessoa não respondeu a questão. Este resultado mostra que o ambiente virtual não passa a sensação de realidade.

Com relação ao envolvimento, a resposta à questão "Sentia-me envolvido pela reprodução 3D da sala daquela época" revelou que $73,2 \%$ dos respondentes se sentiram envolvidos pelo ambiente virtual e $26,8 \%$, indiferentes. Nenhum dos participantes responderam o contrário, mostrando que o ambiente virtual 3D transmite a sensação de envolvimento na época retratada.

A questão "Demorei para perceber que havia uma reprodução 3D por trás" que avaliou novamente a sensação de imersão mostrou que $22 \%$ dos participantes demoraram para perceber que estavam inseridos num ambiente $3 \mathrm{D}$, enquanto que $58,5 \%$ perceberam que havia uma reprodução 3D por trás e 19,5\% foram indiferentes.

Pelos resultados das respostas, percebe-se que mais de $70 \%$ dos participantes tiveram a sensação de estar fisicamente presentes no ambiente virtual tridimensional, além de se sentirem envolvidos durante a interação. A sensação de imersão foi percebida por mais de $50 \%$ dos usuários, mas houveram cerca de $20 \%$ que discordaram desta sensação. Quanto ao grau de realismo quase 20\% discordaram desta sensação. Embora cerca de $25 \%$ afirmaram que a experiência foi realista, pode-se afirmar que o ambiente não passa esta sensação (Figura 3).

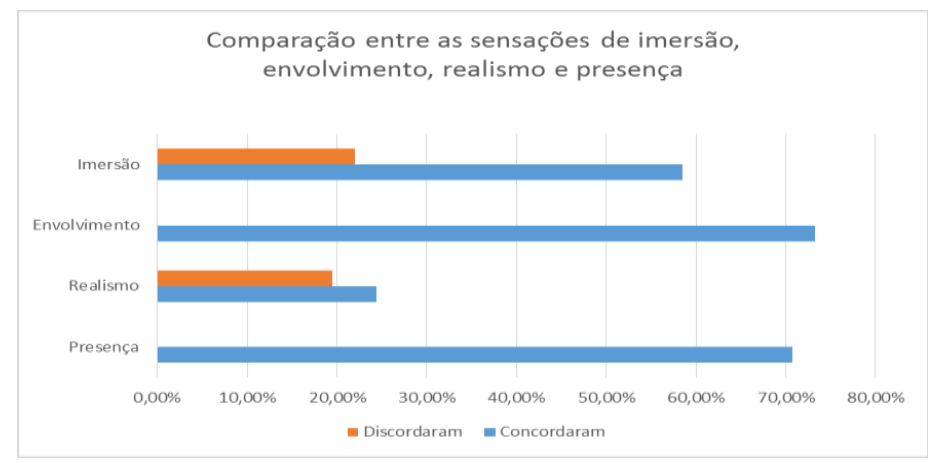

Figura 3. Gráfico comparativo entre as sensações de imersão, envolvimento, realismo e presença

Quanto à navegação e manipulação dos objetos no ambiente virtual tridimensional, 66,7\% dos respondentes acharam a navegação fácil e $30,5 \%$ ficaram indiferentes. Mas houveram 2,8\% que consideram este aspecto difícil.

Para verificar se as informações textuais que apareciam durante a interação complementavam a visualização $3 \mathrm{D}$ com clareza e eficiência, os participantes responderam a questão "A informação textual esclareceu a história do edifício?". Do total, $68,3 \%$ dos participantes concordaram que a informação esclareceu a história do edifício, embora $31,7 \%$ responderam indiferentes. Ninguém respondeu que a informação foi pouco esclarecedora.

Para aferir a satisfação dos usuários com a experiência de interagir com o ambiente virtual tridimensional, foram feitas cinco escalas de qualidade: péssima excelente, frustrante - satisfatória, enfadonho - estimulante, difícil - fácil, rígida flexível. Em relação as qualidades "Péssima" e "Excelente", 73\% dos participantes consideraram a experiência "Excelente", 27\% responderam indiferentes e ninguém 
considerou a experiência péssima. Quanto as qualidades "Frustrante" e "Satisfatória", 70,3\% consideraram a experiência "Satisfatória", 29,7\% responderam indiferentes e ninguém respondeu que a experiência foi frustrante. Nas qualidades "Enfadonho" e "Estimulante", 80,5\% dos participantes consideraram a experiência "Estimulante", $19,5 \%$ responderam indiferentes e ninguém considerou a experiência enfadonha. Nas qualidades "Difícil" e "Fácil", 75\% dos participantes consideraram a experiência "Fácil", 25\% se disseram indiferentes e ninguém avaliou a experiência difícil. Quanto às qualidades "Rígida" e "Flexível", 77,1\% dos participantes consideraram a experiência "Flexível", 20\% foram indiferentes e 2,9\% consideraram a experiência rígida.

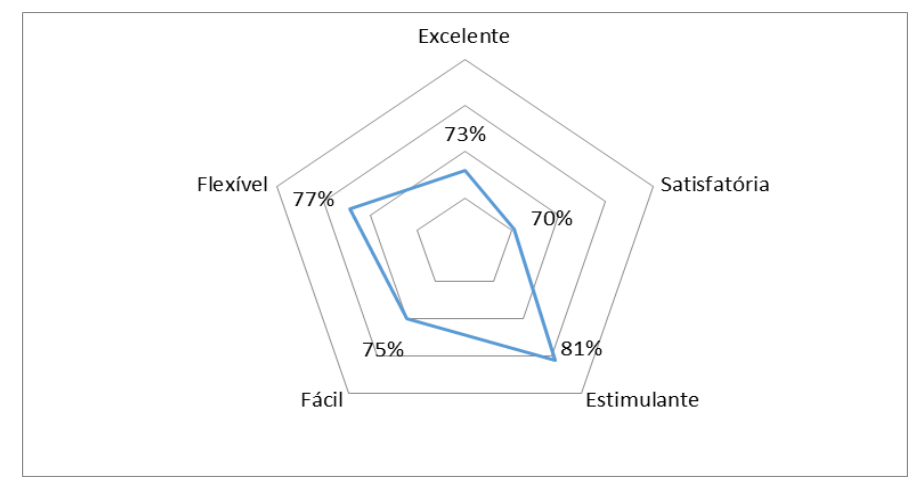

Figura 4. Percentual da satisfação geral dos participantes

Quanto à satisfação, tendo em vista que mais de $70 \%$ dos usuários responderam positivamente em todas as cinco qualidades e apenas uma das qualidades, rígida flexível recebeu $3 \%$ de resposta negativa, pode-se afirmar, que em geral, os participantes se sentiram satisfeitos com a experiência no ambiente virtual tridimensional (Figura 4).

\section{Discussão}

Os ambientes virtuais tridimensionais, quando aplicado a reconstruções do espaço arquitetônico, constituem numa ferramenta que auxilia a exploração e a visualização do espaço. Possuem a flexibilidade de estabelecer relações espaciais e temporais o que leva a um melhor entendimento da tridimensionalidade do volume, seus componentes e as transformações que ocorrem devido ao passar do tempo. Também permitem a inserção de descrições semânticas que estabelecem relações com os valores históricos, sociais, culturais e econômicos do objeto reconstituído.

As sensações de presença, envolvimento e imersão permitem uma exploração do espaço mais próxima do real se comparada com as representações tradicionais de volumetria. Estes ambientes digitais têm se mostrado propícios para estudos do espaço arquitetônico e reconstruções de sítios arqueológicos, que melhoram o entendimento das articulações urbanas do passado.

Devido a estas características os ambientes virtuais tridimensionais com interação e visualização espaço-temporal complementados com descrições semânticas oferecem uma maneira mais interativa de compreender a arquitetura. Podem dar suporte à análise da produção arquitetônica e urbanística ocorridas em diferentes períodos e às relações com os aspectos de ordem social, econômica, política e cultural, questões estas, 
abordadas em disciplinas teóricas do curso de arquitetura, como a Teoria e História da Arquitetura.

Outro aspecto que pode ser explorado pelos ambientes virtuais tridimensionais são as atividades colaborativas envolvidas no processo da produção arquitetônica. Estes ambientes podem tornar-se colaborativos, facilitando a comunicação a distância entre estudantes e docentes.

\section{Considerações Finais}

Este artigo discutiu o potencial dos ambientes virtuais tridimensionais com interações espaço-temporais complementados com descrições semânticas. Mostrou algumas particularidades dos cursos de arquitetura, que envolve habilidades de criação e compreensão do espaço arquitetônico e análises de ordem social, econômica, política e cultural da arquitetura. Descreveu a elaboração de um ambiente virtual tridimensional associado a uma ontologia responsável pelas relações espaço-temporais e acréscimo das descrições semânticas. Apresentou uma avaliação realizada com usuários, para aferir as sensações de presença, imersão, envolvimento e realidade, a eficiência de comunicação das informações semânticas e a usabilidade do ambiente virtual.

A avaliação com os usuários mostrou que o ambiente virtual tridimensional dá a sensação de presença, envolvimento e imersão, o que melhora a compreensão do espaço. A navegação espaço-temporal e a manipulação dos objetos virtuais se mostraram fáceis e as inserções textuais auxiliaram na complementação do entendimento dos valores históricos relacionados ao objeto virtual. Os usuários se sentiram satisfeitos e não encontraram dificuldades durante a interação.

Estes aspectos favorecem o uso dos ambientes virtuais tridimensionais com interações espaço-temporais complementados com descrições semânticas como ferramenta de ensino-aprendizagem nas disciplinas teóricas do curso de arquitetura. Ainda facilitam a comunicação a distância incentivando a realização de trabalhos colaborativos.

Como trabalhos futuros sugere-se uma pesquisa de avaliação dos estudantes de arquitetura utilizando ambientes virtuais tridimensionais como ferramenta de aprendizado em situações do ensino formal das disciplinas teóricas. Esta avaliação deve ser estendida aos docentes para aferir a eficiência de seu uso no processo ensinoaprendizagem.

\section{Referências}

Antoniou, G. e Van Harmelen F. (2008). A Semantic Web Primer. Cambridge: The MIT Press.

Behr, J., Eschler, P., Jung, Y. e Zöllner, M. (2009) "X3DOM - A DOM-based HTML5/ X3D Integration Model", In: 14th International Conference on 3D Web Technology, 2009, Darmstadt. Proceedings... New York: ACM, p.127-135.

Bernardes, P. J. C. (2002). Arqueologia urbana e ambientes virtuais: um sistema para Bracara Augusta. Dissertação (Mestrado em Arqueologia) - Instituto de Ciências Sociais, Universidade do Minho, Braga. Disponível em: $<$ http://repositorium.sdum.uminho.pt/bitstream/1822/191/1/tesepb_vers_final.pdf $>$. Acesso em: 10 dez. 2011. 
Breitman, K. K., Casanova, M. A. e Truszkowski, W. (2007). Semantic web - concepts, technologies and applications. London: Springer.

Cabral, M., Zuffo, M., Ghirotti, S., Belloc, O., Nomura, L., Nagamura, M., Andrade, F., Faria, R. e Ferraz, L. (2007). An experience using X3D for virtual cultural heritage. In: INTERNATIONAL CONFERENCE ON 3D WEB TECHNOLOGY, 12., 2007, Perugia. Proceedings...[S.1.: s.n.], p. 161-164.

Eliseo, M. A. (2012). Modelo de representação espaço-temporal em reconstruções digitais tridimensionais. Tese (Doutorado em Informática) - Instituto Tecnológico de Aeronáutica, São José dos Campos.

Eliseo, M. A., Oliveira, J. M. P. E Pellegrino, S. R. M. (2011). Domain ontology with temporal descriptions for architectural buildings as a support for learning history of architecture. IEEE Multidisciplinary Engineering Education Magazine, v.6, no. 2, p.24 - 31.

Frischer, B. (2005). New directions for cultural virtual reality: a global strategy for archiving, serving, and exhibiting 3D computer models of cultural heritage sites. 2005. Disponível em: $<$ http://www.romereborn.virginia.edu/VR_Frischer2005.pdf $>$. Acesso em: 15/06/2007.

Guarino, N. (1998), "Formal ontology and information systems", International Conference On Formal Ontologies In Information Systems 1998, p. 3-15, Trento.

Gutiérrez, M. A., Vexo, F. e Thalmann, D. (2008). Stepping into virtual reality. London: Springer-Verlag.

Harper, B. D. e Norman, K. L. (1993). Improving user satisfaction: The questionnaire for user interaction satisfaction version 5.5. Proceedings of the 1st Annual Mid-Atlantic Human Factors Conference, 224-228.

Igroup Project Consortium (2008). Igroup Presence Questionnaire (IPQ) overview. Disponível em: <http://www.igroup.org/pq/ipq/>. Acesso em: Setembro 2014.

Maze, J. (2002). Virtual Tactility:Working to Overcome Perceptual and Conceptual Barriers in the Digital Design Studio, in: Proctor, G., ed., Thresholds - Design, Research, Education and Practice, in the Space Between the Physical and the Virtual, 2002. ACADIA Conference Proceedings, Pomona, California, pp. 325-331.

Mobach, M. P. (2008). Do virtual worlds create better real worlds? Virtual Reality. Berlin: Springer.

Rome Reborn. (2010). Site oficial. Virginia. Disponível em: $<\mathrm{http} / / /$ www.romereborn.virginia.edu/>. Acesso em: 20/04/2011.

Roussou, M. (2000). Immersive interactive virtual reality in the museum. Athens: Foundation of the Hellenic World. Disponível em: <http://ui4all.ics.forth.gr/i3SD2000/Roussou.PDF>. Acesso em: 26/05/2007.

São Paulo (Estado). Secretaria de Cultura. Conselho de Defesa do Patrimônio Histórico, Arqueológico, Artístico e Turístico. Resolução SC-27, de 15de dezembro de 1993. Diário Oficial do Estado de São Paulo, Poder Executivo, São Paulo, 16 dez 1993.

Schnabel, M. A. e Kvan, T. (2003). Spatial understanding in immersive virtual environments. In International Journal of Architectural Computing, vol. 1, pp. 435-448.

Web3D Consortium. (2011). X3D overview. Disponível em: $<$ http://www.web3d.org/x3d/specifications/x3d/>. Acesso em: 20/01/2012.

Zevi, B. (2002). Saber ver a arquitetura, São Paulo: Martins Fontes. 